\title{
Comorbilidades neurológicas y su relación con la velocidad de la marcha en adultos mayores del Centro Médico Naval "Cirujano Mayor Santiago Távara” 2010-2015.
}

Neurological comorbidities and its association with gait speed in older adults of the Naval Medical Center "Cirujano Mayor Santiago Távara" 2010-2015.

Karla M. Calderón-Campos ${ }^{1, a}$, José F. Parodi ${ }^{2, b}$, Fernando M. Runzer-Colmenares ${ }^{1,2, b}$

\section{RESUMEN}

Objetivo: Determinar la relación entre presencia de comorbilidades neurológicas y velocidad de la marcha en adultos mayores del Centro Médico Naval "Cirujano Mayor Santiago Távara" 2010-2015. Material y Métodos: Estudio observacional retrospectivo mediante análisis secundario de base de datos. La muestra fue conformada por 1785 adultos mayores. Para determinar la relación entre variables se utilizó Chi-cuadrado con un nivel de significación del 5\% y el programa STATA v.14. Resultados: El 18,7\% de los probandos presentaron enfermedad de Parkinson, 44,9\% presentaron deterioro cognitivo leve y sólo el 3,1\% presentó evidencia de enfermedad cerebrovascular. Existieron diferencias significativas respecto a la edad $(\mathrm{p}<0,001)$ y sexo $(\mathrm{p}=0,043)$ según la velocidad de marcha (mayor en mujeres de 80 años o más). Hubo relación significativa entre el deterioro cognitivo leve $(\mathrm{p}<0,001)$ y la enfermedad cerebro- vascular $(\mathrm{p}=0,002)$ con la velocidad de marcha. La probabilidad de una velocidad de marcha lenta ajustada por antecedente de enfermedad de Parkinson, edad y sexo para aquellos con deterioro cognitivo leve fue de 2,13 (IC95\% 1,72-2,63) y para aquellos con enfermedad cerebrovascular fue de 1,79 (IC95\% 1,01-3,20). Conclusiones: La lentitud de marcha se presentó mayormente en mujeres de 80 o más años de edad y se relacionó significativamente con el deterioro cognitivo leve y enfermedad cerebrovascular, pero no con la enfermedad de Parkinson.

PALABRAS CLAVE: Trastornos neurocognitivos, velocidad de marcha, adultos mayores, enfermedad de Parkinson, enfermedad cerebro-vascular, demencia.

\section{SUMMARY}

Objective: To determine the association between presence of neurological comorbidities and walking speed in older adults attended at the Naval Medical Center "Cirujano Mayor Santiago Távara" 2010-2015. Methods: Observational retrospective study through secondary analysis of a database of 1785 older adults. To determine the relationship between variables, the Chi-square test with a level of significance of 5\%, and the STATA v.14 program were used. Results: $18.7 \%$ of the probands had Parkinson's disease, $44.9 \%$ had mild cognitive impairment,

1 Universidad Científica del Sur. Lima, Perú.

2 Universidad de San Martín de Porres, Facultad de Medicina Humana, Centro de Investigación del

Envejecimiento (CIEN). Lima, Perú.

a Estudiante de Medicina Humana.

b Médico Geriatra. 
Comorbilidades neurológicas y su relación con la velocidad de la marcha en adultos mayores.

and only $3.1 \%$ had cerebrovascular disorder. There were significant differences in walking speed with respect to advanced age and female sex (greater in 80 y.o. or older females). There was a significant relationship between mild cognitive impairment and cerebrovascular disorder with walking speed. The probability of a slow walking speed adjusted for Parkinson's disease diagnosis, age and sex for those with mild cognitive impairment was 2.13 (IC95\% 1.72-2.63), and for those with cerebrovascular disease, 1.79 (IC95\% 1.01-3.20). Conclusions: Slow walking was presented mainly in women over 80 years of age, and was significantly related to mild cognitive impairment and cerebrovascular disorder, but not to Parkinson's disease

KEY WORDS: cognitive impairment, gait speed, elderly, Parkinson's disease, stroke, dementia.

\section{INTRODUCCIÓN}

La velocidad de la marcha es un indicadorimportante de las capacidades funcionales de las personas adultas mayores. La asociación entre edad y enlentecimiento de la marcha se explicarían por los diversos cambios a nivel fisiológico y estructural que se dan con el incremento de la edad (1). Se ha demostrado que la reducción de la velocidad de la marcha se asocia con un mayor riesgo de caídas, discapacidad, dependencia, hospitalización y mortalidad, tanto en personas mayores frágiles como en buen estado de salud. (26) Las causas de la disminución de la velocidad de la marcha en las personas mayores no están claras, pero se incluyen afecciones neurológicas (p. ej., demencia, depresión y/o ansiedad o alteraciones sensoriales o motoras) y no neurológicas (p. ej., sarcopenia, osteoartritis y factores de riesgo cardiovascular) (5,79). De igual modo, ciertas enfermedades neurológicas propias de la edad avanzada, como la enfermedad de Parkinson, pueden causar una disminución de la velocidad de la marcha y alteraciones de la misma (10). Por su naturaleza, el envejecimiento incrementa el riesgo de padecer afecciones neurocognitivas, las cuales se consideran como una de las principales razones de discapacidad en los adultos mayores (11). Diversos estudios han sugerido una interrelación entre las deficiencias cognitivas y los trastornos de movilidad, incluida la reducción de la velocidad de la marcha. De la misma forma, las bases biológicas de esta relación han sido objeto de estudio. Holtzer et al. han demostrado que la marcha y la función cognitiva se asocian con cambios estructurales subyacentes compartidos en los lóbulos temporales prefrontal y medial. (12) Los estudios existentes proporcionan evidencia de que las personas con deterioro cognitivo leve pueden experimentar disfunción de la velocidad de la marcha (13-16). Además, estudios han indicado que la velocidad más lenta de la marcha habitual predecirá a su vez el declive cognitivo acelerado en los adultos mayores $(17,18)$. Por lo tanto, existe evidencia suficiente de la relación entre la velocidad de la marcha y la cognición en el envejecimiento. A nivel nacional son pocos los estudios que hacen referencia a las comorbilidades neurológicas y su relación con la velocidad de la marcha $(1,15)$.

Mediante el presente estudio se buscó determinar la relación entre el cambio en la velocidad de la marcha y la presencia de comorbilidades neurológicas en la población adulto mayor atendida en el nosocomio en mención durante el periodo 2010-2015.

\section{MATERIAL Y MÉTODOS}

El estudio fue observacional, analítico, retrospectivo, análisis secundario de base de datos (los datos que se utilizaron para este estudio provienen de una base de datos); en el estudio original muchos de los datos se tomaron del paciente y los otros mientras se les evaluaba, se hacía la revisión de su historia. La población del estudio original estuvo conformada por 1897 pacientes adultos mayores ( $\geq 60$ años), atendidos en el Servicio de Geriatría del Centro Médico Naval "Cirujano Mayor Santiago Távara" durante los años 2010 al 2015. En el estudio original todos los participantes provenían de los niveles asistenciales ambulatorios (consultorios externos y clínica de día) residían en Lima o Callao, Perú, la mayoría de ellos eran hombres y militares retirados mientras que los participantes restantes consistían en sus familiares; se creó una base de datos con todos los participantes elegibles y se contactó con ellos por teléfono para invitarlos a participar (19). El objetivo del estudio madre fue explorar los factores asociados a fragilidad en adultos mayores.

Para el presente estudio, se conformaron 2 grupos: adultos mayores con velocidad de marcha lenta y adultos mayores con velocidad de marcha normal. Se excluyeron a los pacientes geriátricos con patología traumática en miembros inferiores y con antecedente de demencia establecida, es decir, con diagnóstico sindrómico de demencia por examen clínico y 
documentada por tests psicológicos. De igual forma se excluyó a pacientes con dificultad para realizar pruebas cognitivas, con problemas auditivos y/o visuales severos, con otros problemas físicos que pudieran interferir con su performance, con lengua diferente al español, aquellos catalogados con pseudodemencia, con diagnóstico de depresión y rendimiento cognitivo bajo por uso de fármacos como biperideno; criterios definidos por Custodio (20). Se eliminaron los datos de los participantes con puntaje $<17$ en el Mini Mental State Examination (MMSE). Finalmente, se evaluaron a 1785 adultos mayores para alcanzar el objetivo del estudio.

La velocidad de la marcha se definió como el movimiento rítmico y la posibilidad de deambular en 2 pies. Teniendo en cuenta ambos componentes, se consideró marcha lenta cuando la velocidad fue $\leq 0,8$ metros por segundo (21).

Las comorbilidades neurológicas evaluadas fueron enfermedad de Parkinson, deterioro cognitivo leve, enfermedad cerebro vascular, patologías escogidas por los investigadores principales por ser comorbilidades frecuentes en el adulto mayor y las que mas frecuentemente se encontraron en las historias clínicas.

El deterioro cognitivo leve, una condición neurológica común durante envejecimiento, fue determinado por el hallazgo de un puntaje entre 23 y 25 en la escala de Montreal Cognitive Assessment (MoCA) (22). Tanto la enfermedad de Parkinson, una enfermedad neurológica degenerativa y progresiva debido a la disminución de neuronas dopaminérgicas y caracterizada por la presencia de temblores, rigidez muscular y alteración de los reflejos posturales $(23,24)$, como la enfermedad cerebro vascular, entendido como déficit neurológico producto de la rotura de un vaso sanguíneo o el bloqueo del flujo sanguíneo por un coágulo con consecuente muerte neuronal debido a la hipoxia (25), fueron identificados como variables de estudio por su registro en la historia clínica del paciente; para el primer caso los pacientes con enfermedad de Parkinson se encontraban entre los estadios 1 y 3 de la escala de Hoehn \& Yahr mientras que los pacientes con enfermedad cerebro vascular, tenían alguna secuela funcional pero ninguno con dependencia total, todos podían acudir a consultorio con algún grado de independencia.

Se recurrieron a fuentes secundarias de información, se revisó la base de datos de los pacientes adultos mayores atendidos en el nosocomio de estudio. Los datos se recogieron mediante una ficha de recolección. Para el análisis estadístico se utilizó el programa estadístico STATA v.14 y se aplicó la prueba Chi-cuadrado con un nivel de significancia del 5\% para estimar la relación entre variables. Finalmente, se construyó un modelo de regresión logística para determinar la asociación entre las comorbilidades neurológicas en estudio y la velocidad de marcha lenta, incluyendo en el modelo final a las variables significativas en el análisis bivariado.

La información de estudio fue obtenida con el consentimiento de las autoridades del Centro Médico Naval "Cirujano Mayor Santiago Távara", siendo esta socializada al personal de salud correspondiente, así como al Comité de ética de la Universidad Cientifica del Sur. Sumado a ello no se registraron datos personales de los pacientes, a cada uno se le asignó un código para poder identificarlos. La tabla 1 corresponde al análisis descriptivo univariado donde se presentan las frecuencias absolutas y porcentuales de los datos generales, en las tablas 2 y 3 se presenta el análisis bivariado donde se analiza la relación entre los datos generales y la velocidad de marcha mediante la prueba Chi-cuadrado, y en la tabla 4 se presenta el análisis de regresión logística para analizar la asociación entre las comorbilidades neurológicas y la velocidad de marcha lenta.

\section{RESULTADOS}

Durante el periodo 2010-2015 se atendieron un total de 1896 adultos mayores en el Centro Médico Naval "Cirujano Mayor Santiago Távara". Sin embargo, para el presente estudio se excluyeron a aquellos que no cumplieron los criterios de selección, la población estudiada quedó conformada por 1785 adultos mayores. Entre las características generales de los adultos mayores (tabla 1), el 45,9\% tenía entre 71 a 80 años de edad, el 58,3\% fue del sexo masculino y el $65,4 \%$ tenía estudios escolares completos/incompletos. Respecto a las comorbilidades neurológicas, el 18,7\% presentaron enfermedad de Parkinson, el 44,9\% presentaron deterioro cognitivo leve y sólo el 3,1\% de los adultos mayores presentaron enfermedad cerebro vascular. A su vez, la velocidad de marcha del 34,8\% de participantes fue alterada o lenta, es decir, inferior a $\operatorname{los} 0,8 \mathrm{~m} / \mathrm{s}$.

Existieron diferencias significativas respecto a la edad según la velocidad de marcha (tabla 2). Se evidenció que el $58,8 \%$ de los pacientes con velocidad de marcha alterada (lenta) eran mayores de 80 años, 
Tabla 1. Características generales de los adultos mayores del Centro Médico Naval "Cirujano Mayor Santiago Távara" 2010-2015.

\begin{tabular}{lcc}
\hline \multicolumn{1}{c}{ Datos generales } & $\mathbf{n}=\mathbf{1 7 8 5}$ & $\mathbf{\%}$ \\
\hline Edad & 281 & $15,7 \%$ \\
$\quad 60$ - 70 años & 820 & $45,9 \%$ \\
71 - 80 años & 684 & $38,3 \%$ \\
$\quad>80$ años & & \\
Sexo & \\
$\quad$ Femenino & 740 & $41,5 \%$ \\
$\quad$ Masculino & 1040 & $58,3 \%$ \\
Grado de instrucción ${ }^{1}$ & & \\
$\quad$ Técnico/superior & 515 & $28,9 \%$ \\
$\quad$ Colegio completo/incompleto & 1168 & $65,4 \%$ \\
Enfermedad de Parkinson & & \\
$\quad$ Alterado & 333 & $18,7 \%$ \\
$\quad$ Normal & 1452 & $81,3 \%$ \\
Deterioro cognitivo leve & & \\
$\quad$ Sí & 801 & $44,9 \%$ \\
$\quad$ No & 984 & $55,1 \%$ \\
Enfermedad cerebro vascular & & \\
$\quad$ Sí & 55 & $3,1 \%$ \\
$\quad$ No & 1730 & $96,9 \%$ \\
Velocidad de la marcha & & \\
$\quad$ Alterada & \\
$\quad$ Normal & 621 & $34,8 \%$ \\
\hline${ }^{1}$ Los datos no podrían sumar 1785 debido a datos faltantes, en \\
ningún caso supero al 10\%.
\end{tabular}

mientras que sólo el $27,4 \%$ de aquellos con velocidad de marcha normal pertenecían al mismo grupo etario. Asimismo, existieron diferencias significativas respecto a la velocidad de marcha según sexo del paciente. Se observó que el 44,8\% del grupo que tenía velocidad de marcha lenta eran del sexo femenino, proporción superior al 39,8\% de las mujeres mayores con velocidad de marcha normal. El análisis bivariado demostró que hubo relación significativa entre el deterioro cognitivo leve y la enfermedad cerebro vascular con la velocidad de marcha (tabla 3). El $57,8 \%$ de aquellos con velocidad de marcha lenta presentaron deterioro cognitivo leve, mientras que sólo el 38\% de los pacientes con velocidad de marcha normal presentaron dicha comorbilidad neurológica. Del mismo modo, el 4,8\% de los adultos mayores con velocidad de marcha lenta presentaron enfermedad cerebro vascular, proporción superior al 2,1\% de los participantes con velocidad de marcha normal que presentaron dicho desorden neurológico. Por otro lado, la enfermedad de Parkinson no se relacionó significativamente con la velocidad de marcha. Se observó que el $19,8 \%$ y $18 \%$ de los adultos mayores con velocidad de marcha lenta y normal, respectivamente, presentó enfermedad de Parkinson. En el análisis de regresión logística para determinar factores asociados a velocidad de marcha lenta independientemente del antecedente de enfermedad de Parkinson, edad y sexo, se pudo apreciar que el deterioro cognitivo leve aumenta 2,13 veces la probabilidad de desarrollar velocidad de marcha lenta y la enfermedad cerebrovascular la aumenta 1,79 veces (tabla 4 ).

Tabla 2. Características generales según velocidad de la marcha de los adultos mayores del Centro Médico Naval “Cirujano Mayor Santiago Távara” 2010-2015.

\begin{tabular}{|c|c|c|c|c|c|}
\hline \multirow{3}{*}{ Datos generales } & \multicolumn{4}{|c|}{ Velocidad de la marcha } & \multirow{3}{*}{$\mathbf{p}^{2}$} \\
\hline & \multicolumn{2}{|c|}{ Alterada } & \multicolumn{2}{|c|}{ Normal } & \\
\hline & $n=621$ & $\%$ & $n=1164$ & $\%$ & \\
\hline \multicolumn{6}{|l|}{ Edad } \\
\hline $60-70$ años & 52 & $8,4 \%$ & 229 & $19,7 \%$ & \multirow{3}{*}{$<0,001$} \\
\hline 71 - 80 años & 204 & $32,9 \%$ & 616 & $52,9 \%$ & \\
\hline$>80$ años & 365 & $58,8 \%$ & 319 & $27,4 \%$ & \\
\hline \multicolumn{6}{|l|}{$\operatorname{Sexo}^{1}$} \\
\hline Femenino & 277 & $44,8 \%$ & 463 & $39,8 \%$ & \multirow{2}{*}{0,043} \\
\hline Masculino & 341 & $55,2 \%$ & 699 & $60,2 \%$ & \\
\hline \multicolumn{6}{|l|}{ Grado de instrucción ${ }^{1}$} \\
\hline Técnico/superior & 189 & $33,5 \%$ & 326 & $29,1 \%$ & \multirow{2}{*}{0,066} \\
\hline Colegio completo/incompleto & 375 & $66,5 \%$ & 793 & $70,9 \%$ & \\
\hline
\end{tabular}


Tabla 3. Comorbilidad neurológica según velocidad de la marcha de los adultos mayores del Centro Médico Naval "Cirujano Mayor Santiago Távara” 2010-2015.

\begin{tabular}{|c|c|c|c|c|c|}
\hline \multirow{3}{*}{ Comorbilidad neurológica } & \multicolumn{4}{|c|}{ Velocidad de la marcha } & \multirow{3}{*}{$\mathbf{p}^{1}$} \\
\hline & \multicolumn{2}{|c|}{ Alterada } & \multicolumn{2}{|c|}{ Normal } & \\
\hline & $n=621$ & $\%$ & $n=1164$ & $\%$ & \\
\hline \multicolumn{6}{|l|}{ Enfermedad de Parkinson } \\
\hline Alterado & 123 & $19,8 \%$ & 210 & $18,0 \%$ & \multirow{2}{*}{0,362} \\
\hline Normal & 498 & $80,2 \%$ & 954 & $82,0 \%$ & \\
\hline \multicolumn{6}{|l|}{ Deterioro cognitivo leve } \\
\hline Sí & 359 & $57,8 \%$ & 442 & $38,0 \%$ & \multirow{2}{*}{$<0,001$} \\
\hline No & 262 & $42,2 \%$ & 722 & $62,0 \%$ & \\
\hline \multicolumn{6}{|l|}{ Enfermedad cerebro vascular } \\
\hline Sí & 30 & $4,8 \%$ & 25 & $2,1 \%$ & \multirow{2}{*}{0,002} \\
\hline No & 591 & $95,2 \%$ & 1139 & $97,9 \%$ & \\
\hline
\end{tabular}

Tabla 4. Regresión logística: Asociación entre Comorbilidades neurológicas y velocidad de marcha lenta $(\mathrm{n}=1785)$

\begin{tabular}{lc}
\hline \multicolumn{1}{c}{ Variables } & OR (IC al 95\%) \\
\hline Deterioro cognitivo leve & $2,13(1,72-2,63)$ \\
Enfermedad Cerebrovascular & $1,79(1,01-3,20)$ \\
\hline${ }^{1}$ OR: Odds Ratio // IC: Intervalo de confianza &
\end{tabular}

\section{DISCUSIÓN}

En esta muestra de 1785 adultos mayores atendidos en el Centro Médico Naval "Cirujano Mayor Santiago Távara”, la velocidad de la marcha más lenta se asoció significativamente, en el modelo ajustado, con el diagnostico de deterioro cognitivo leve y enfermedad cerebro vascular, mas no con la Enfermedad de Parkinson. Un adulto mayor con deterioro cognitivo leve o diagnóstico de enfermedad cerebro vascular tuvo una mayor probabilidad de presentar velocidad de marcha lenta, con un OR ajustado de 2,13 y 1,79 respectivamente (riesgo independiente ajustado por antecedente de enfermedad de Parkinson, edad y sexo). Este hallazgo está en línea con otros estudios peruanos, como el de Rodríguez et al, y Parodi et al., donde se encontró que la marcha lenta $(<$ de $1 \mathrm{~m} / \mathrm{s}$ o $<$ de $0,8 \mathrm{~m} / \mathrm{s}$, respectivamente) se relacionó con la presencia de trastornos neurocognitivos $(15,16)$. De la misma forma con lo reportado en Japón por Doi et al. y la revisión sistemática de Bahureksa at al., $(13,14)$, ambos estudios demostraron que el deterioro cognitivo leve afecta la velocidad de la marcha significativamente. Por otro lado, en el estudio actual se encontró mayor incidencia de velocidad de marcha más lenta a edades mayores, siendo esta relación significativa. También se observó que las mujeres y los hombres difieren significativamente en cuanto a los patrones de velocidad de la marcha, donde hubo mayor incidencia de velocidad de la marcha alterada entre las mujeres estudiadas. Esto coincide con lo informado por Rosas et al., quienes evidenciaron que la edad y la velocidad de la marcha se relacionaban de forma inversa y significativa, es decir, a más edad menor velocidad de marcha; de la misma forma observaron diferencias entre el promedio de la velocidad de marcha entre mujeres y hombres $(0,71 \mathrm{~m} / \mathrm{s}$. y $0,85 \mathrm{~m} / \mathrm{s}$ respectivamente) (26).

Los hallazgos de la presente investigación sugieren que la velocidad de la marcha lenta en los adultos mayores podría ser reflejo de cambios a nivel cerebral, específicamente debido a trastornos neurológicos clínicos y subclínicos como deterioro cognitivo leve y enfermedad cerebro vascular; lo cual podría estar influenciado a su vez por la edad y género del adulto mayor. Esto destaca la importancia de la evaluación e identificación temprana de este tipo de trastornos que puede prevenir diversos resultados adversos producto de la disminución de la velocidad de la marcha, la misma que afecta en gran medida la calidad de vida de los adultos mayores, lo que podría 
ser un argumento para las medidas de prevención y corrección. Es particularmente interesante que la alteración de la velocidad de la marcha no esté asociada con la enfermedad de Parkinson, teniendo en cuenta que los trastornos de la marcha constituyen uno de los síntomas más comunes en dicha condición (27). Sumado a ello, los metanálisis revelan que en las personas con Enfermedad de Parkinson que tienen más riesgo de caída, hay una velocidad de marcha más lenta, cadencia más baja, pasos más cortos y más movimiento mediolateral de la cabeza y la pelvis (28). Una posible explicación a la discordancia encontrada, es que durante el estudio no se tomó el tipo de calzado que usaron las personas lo cual puede influir en el resultado de las características de la marcha de las personas con enfermedad de Parkinson (29). Se necesitan de más estudios al respecto.

Entre las principales limitaciones, es importante mencionar que la población es de origen hospitalario y la frecuencia de comorbilidades podría ser mayor a la de la población general, además al ser un Centro Médico Militar, donde se atienden marinos y sus familiares, estos presentan características sociodemográficas distintas. Se debe considerar también, que los participantes con enfermedad de Parkinson son parte de un programa de reacondicionamiento físico, lo que podría haber sesgado los resultados. Finalmente, la información recolectada será de utilidad solo para la realidad del nosocomio donde se realizó la investigación.

Se concluye que la velocidad de marcha lenta se presentó mayormente en adultos varones con edad $>80$ años y se relacionó significativamente con el deterioro cognitivo leve y la enfermedad cerebro vascular, pero no con la enfermedad de Parkinson. Cabe señalar que esta última comorbilidad neurológica fue más frecuente que la enfermedad cerebro vascular pero menos que el deterioro cognitivo leve. Estos resultados ponen en relevancia el planteamiento de políticas públicas para la generación de acciones preventivas con vistas a mejorar la calidad de vida de las personas mayores. Se propone la implementación sistemática de tamizajes de enfermedad neurológica en geriatría y la evaluación de la velocidad de la marcha.

\section{Declaración de Financiamiento y de Conflictos de Interés}

El presente estudio fue una iniciativa de investigación autofinanciada. Los autores contaron con los suficientes recursos humanos, económicos y materiales para poder llevar a cabo la investigación desde su planificación hasta su culminación. No se ocasionaron gastos a las instituciones involucradas. De igual forma no hubo algún tipo de conflicto de interés.

\section{Correspondencia}

Karla Mercedes Calderón-Campos

Jr. Las Acebos \#183 Urb. Las Flores, San Juan de Lurigancho, Lima, Perú

Celular: 51956377730

Correo electrónico: karla_4119@hotmail.com

\section{REFERENCIAS BIBLIOGRÁFICAS}

1. Varela L, Saavedra P, Chavez H. Velocidad de la marcha en adultos mayores de la comunidad en Lima, Perú. Rev Med Hered. 2009; 20(3): 133-138.

2. Kyrdalen I, Thingstad P, Sandvik L, Ormstad H. Associations between gait speed and well-known fall risk factors among community-dwelling older adults. Physiother Res Int. 2018; 10: e1743.

3. Artaud F, Singh-Manoux A, Dugravot A, Tzourio C, Elbaz A. Decline in fast gait speed as a predictor of disability in older adults. J Am Geriatr Soc. 2015; 63(6): 1129-36.

4. Heiland E, Welmer A, Wang R, Santoni G, Angleman $\mathrm{S}$, Fratiglioni L, et al. Association of mobility limitations with incident disability among older adults: a population-based study. Age Ageing. 2016; 45(6): 812-819.

5. Tian Q, Zhang M, Deng Y, Duan $Y, T u$ Q, Cao $\mathrm{Y}$, et al. Does gait speed replace comprehensive geriatric assessment in the elderly? Int $\mathrm{J}$ Gerontol. 2016; 10(4):232-236.doi: http://dx.doi. org/10.1016/j.ijge.2016.03.010

6. Perera S, Patel K, Rosano C, Rubin S, Satterfield S, Harris T, et al. Gait speed predicts incident disability: A pooled analysis. J Gerontol A Biol Sci Med Sci. 2016; 71(1): 63-71.

7. Lipsitz L, Manor B, Habtemariam D, Iloputaife I, Zhou J, Travison T. The pace and prognosis of peripheral sensory loss in advanced age: association with gait speed and falls. BMC Geriatr. 2018; 18(1): 274.

8. Heiland E, Qiu C, Wang R, Santoni G, Liang Y, Fratiglioni L, et al. Cardiovascular risk burden and future risk of walking speed limitation in older adults. J Am Geriatr Soc. 2017; 65(11): 2418-2424.

9. Welmer A, Angleman S, Rydwik E, Fratiglioni L, Qiu C. Association of cardiovascular burden with mobility limitation among elderly people: a population-based study. PLoS One. 2013; 8(5): e65815. 
10. Paker N, Bugdayci D, Goksenoglu G, Demircioğlu D, Kesiktas N, Ince N. Gait speed and related factors in Parkinson's disease. J Phys Ther Sci. 2015; 27(12): 3675-3679.

11. Organización Mundial de la Salud. Demencia. Ginebra: Organización Mundial de la Salud; 2017. (Fecha de acceso 12 de setiembre de 2018). Disponible en: http://www.who.int/es/news-room/fact-sheets/ detail/dementia.

12. Holtzer R, Epstein N, Mahoney J, Izzetoglu M, Blumen $H$. Neuroimaging of mobility in aging: a targeted review. J Gerontol A Biol Sci Med Sci. 2014; 69(11): 1375-88.

13. Doi T, Shimada H, Park H, Makizako H, Tsutsumimoto $\mathrm{K}$, Uemura $\mathrm{K}$, et al. Cognitive function and falling among older adults with mild cognitive impairment and slow gait. Geriatr Gerontol Int. 2015; 15(8): 1073-8.

14. Bahureksa L, Najafi B, Saleh A, Sabbagh M, Coon D, Mohler M, et al. The Impact of Mild Cognitive Impairment on Gait and Balance: A Systematic Review and Meta-Analysis of Studies Using Instrumented Assessment. Gerontology. 2017; 63(1): 67-83.

15. Rodríguez G, Burga D, Cipriano G, Ortiz P, Tello T, Casas P, et al. Factores asociados a velocidad de marcha lenta en adultos mayores de un distrito en Lima, Perú. Rev Peru Med Exp Salud Publica. 2017; 34(4): 619-626.

16. Parodi J, Nieto W, Tellez W, Ventocilla I, Runzer F, Taype A. Velocidad de marcha y desarrollo de trastornos neurocognitivos en adultos mayores: resultados de una cohorte peruana. Rev Esp Geriatr Gerontol. 2017; 53(2): 73-76.

17. Ojagbemi A, D'Este C, Verdes E, Chatterji S, Gureje O. Gait speed and cogni-tive decline over 2 years in the Ibadan study of aging. Gait Posture. 2015; 41(1): 736-740.

18. Beauchet O, Annweiller C, Callisaya M, De Cock A, Helbostad J, Kressig R, et al. Poor gait performance and prediction of dementia: results from a metaanalysis. J Am Med Dir Assoc. 2016; 17(6): 482-490.
19. Runzer-Colmenares F, Samper-Ternent R, Al Snih S, Ottenbacher K, Parodi J, Wong R. Prevalence and factors associated with frailty among Peruvian older adults. Arch Gerontol Geriatr. 2014; 58(1): 1-11.

20. Custodio N, García A, Montesinos R, Escobar J, Bendezú L. Prevalencia de demencia en una población urbana de Lima-Perú: estudio puerta a puerta. An Fac med. 2008; 69(4): 233-8.

21. Cerda L. Manejo del trastorno de marcha del adulto mayor. Rev Med Clin Condes. 2014; 25(2): 265-275.

22. Knopman D, Peterson R. Mild Cognitive impairment and mild dementia: A clinical perspective. Mayo Clin Proc. 2014; 89(10): 1452-1459.

23. Chávez E, Ontiveros M, Carrillo J. La enfermedad de Parkinson: neurología para psiquiatras. Salud Mental. 2013; 36: 315-324.

24. Sarró T. Enfermedad de Parkinson y otros trastornos del movimiento. Madrid, España: Elsevier; 2012.

25. Barrientos M, Yangali V. Efectividad de las intervenciones mediante ejercicio fisico basadas en el hogar en personas de 50 a 75 años de edad con Alzheimer, desorden cerebrovascular y Parkinson. Tesis de grado. Lima, Perú: Universidad Privada Norbert Wiener; 2016.

26. Rosas G, Yarce E, Paredes Y, Rosero M, Morales A. Velocidad de la marcha en ancianos de la comunidad de la ciudad de Pasto. Unimar. 2015; 33(1): 191-199.

27. Gonçalves G, Pereira J. Trastorno de la marcha en la enfermedad de Parkinson: freezing y perspectivas actuales. Rev Med Chile. 2013; 141(6): 758-764.

28. Creaby M, Cole M. Gait characteristics and falls in Parkinson's disease: A systematic review and metaanalysis. Parkinsonism Relat Disord. 2018; 57: 1-8.

29. Pereira M, Orcioli-Silva D, Sousa P, Beretta V, Gobbi L. The effects of habitual footwear in gait outcomes in people with Parkinson's disease. Gait Posture. 2018; 68: 111-114.

Recibido: 23/01/2019

Aceptado: 24/04/2019 